Keywords: arterial; diagnosis; embolization; IVR; shock; trauma; treatment

Prehosp Disast Med 2005;20(3):s145-s146

Comparison of Abdominal X-ray and CT Scan in 56 Opium and Heroin Body Packers in Mashhad, Iran M. Balali-Mood; G. Zare

Mashhad University of Medical Sciences, Iran

Introduction: Opium and heroin body packing is a social and health problem in Mashhad since the city is in the vicinity of the Afghanistan border.

Objective: To study health aspects of the body packers admitted in Imam Reza Toxicology Ward between 2001 and 2004 and to compare abdominal X-ray (AXR) and computed tomography (CT) scan for the diagnosis of the patients.

Methods: All body packers referred to the Toxicology Ward of Imam Reza Hospital in Mashhad, Iran between 2001 and 2003 were studied. They were hospitalized and underwent close observation for any sign of intoxication. An AXR and a urine morphine test were performed for all patients. An abdominal CT scan also was carried out for 41 of these patients. Naloxane and supportive care were applied to those with clinical manifestations of intoxication. AXR and CT scan results were classified into three groups (highly suggestive, suggestive, and false positive) and compared, using the chi-square test.

Results: A total of 56 body packers (54 males and two females) aged $32.1 \pm 11.3$ years were studied. A mean of $44.4 \pm 35.1$ opium and $52.0 \pm 20.0$ heroin packets weighing 8-15 grams were retrieved from 46 and 10 patients, respectively. Most of the patients (43\%) were uneducated, while $32 \%, 23 \%$, and $2 \%$ of them had primary, secondary, and university education, respectively. The majority of the patients $(71 \%)$ were married, but only $25 \%$ of them were employed. The highest frequency of admissions was in autumn (18 patients) followed by winter (17 patients) and summer ( 15 patients). Twenty-six patients were unconscious at the time of admission and six of these were admitted to the intensive care unit (ICU). All unconscious patients responded well to Naloxane. The mean period of hospitalization was $4.7 \pm 2.7$ days for opium and $4.1 \pm 0.6$ days for heroin packers. The urine morphine test was positive in $82 \%$ of the patients. Nine patients underwent surgical operation and death occurred in only three opium packers. As shown in the table, AXR and CT scan results were significantly different $\left(\chi^{2}=46.1\right.$, diff $\left.=2, p<0.001\right)$.

Considering defecation of the packets as the gold standard diagnostic method, sensitivity of AXR and CT scan in diagnosis of body packing was determined as $92 \%$ and $100 \%$, respectively.

Conclusions: Although AXR is a simple and more available technique for the diagnosis of opium and heroin body packing, CT scan was shown to be a more significant sensitive diagnostic tool and should be employed as the confirmatory method.

\begin{tabular}{|l|c|c|}
\hline \multicolumn{1}{|c|}{ Results } & \multicolumn{2}{c|}{ Diagnostic Methods } \\
\hline & Abdominal X-ray & Abdominal CT Scan \\
\hline Highly Suggestive & 16 & 40 \\
\hline Suggestive & 36 & 1 \\
\hline False Negative & 4 & 0 \\
\hline Total & $\mathbf{5 6}$ & $\mathbf{4 1}$ \\
\hline
\end{tabular}

Table 1-Comparison of abdominal X-ray and CT scan results in diagnosis of body packing $(\mathrm{CT}=$ computed tomography)

Keywords: body packing; CT scan; diagnosis; heroin; opium; patients; $x$-ray

Prebosp Disast Med 2005;20(3):s146

\section{Use of Hypnosis in General Emergency Service}

F. van Trimpont

$\mathrm{CH}$ Hornu-Frameries, Belgium

Introduction: Hypnosis can provide an interessant support to analgesia and relaxation of patient in a general emergency service. Indications for and feasibility of using hypnosis in such circumstances are reported.

Methods: Two indications for using hypnosis were selected. First, it can provide help to manage analgesia. Hypnosis generally can be practiced with children before realization of other techniques (IV line, local or regional anesthesia). Children are more relaxed after hypnosis and therefore respond better to medical examinations and techniques. This indication also is useful for anxious adults as a secondary technique to complement analgesia. A second indication of using hypnosis is relaxation for patients who need non-invasive, respiratory techniques. These patients generally have problems breathing into such apparatuses and an easy technique, like hypnosis, which doesn't modify respiratory rates, helps patients accept non-invasive, respiratory techniques and avoid intubation.

Conclusion: The use of hypnosis in an emergency service can help patients accept some techniques used in emergency situations and also can allow patients to be more relaxed and have a better experience with analgesia, especially for children.

Keywords: analgesia; children; emergency services; hypnosis; indications; relaxation

Prehosp Disast Med 2005;20(3):s146

\section{New Criteria for Deciding Damage Control Surgery for Severe Abdominal Trauma}

K. Mashiko; Y. Sakamoto; H. Matsumoto; K. Takubiro; Y. Hara; R. Morita; K. Takei; Y. Ueno; K. Abe; Y. Tomita

Chiba-Hokusoh Hospital, Japan

Purpose: As a standard, factors that determine the need for damage control surgery (DCS) include the deadly triad of acidosis, hypothermia, and coagulopath. However confirmation of the coagulopathy requires time and it might not be possible to utilize the triad in the emergency room (ER) setting. Therefore, the simple and practical criteria for the DCS (hypotension, hypothermia, and acidosis) in victims of severe abdominal trauma were evaluated. 